\title{
MEASUREMENT OF THE DEL ‘YED NEUTRON FRACTION ANN CORRECTION OF SMALL ANGLE SCATTERING DATA FROM A PULSED SPALLATION SOURCE *
}

\author{
J.E. EPPERSON ${ }^{1)}$, J.M. CARPENTER ${ }^{2)}$, P. THIYAGARAJAN ${ }^{2)}$ and B. HEUSER ${ }^{3)}$ \\ 1) Materials Science Division, Argonne National Laboratory, Argonne, IL 60439, USA \\ 2) Intense Pulsed Neutron Source Division, Argonne National Laboratory, Argonne, IL 60439, USA \\ 3) Nuclear Engineering Department, University of Michigan, Ann Arbor, MI 48109, USA
}

Received 23 October 1989

The fraction of delayed neutrons in a moderated beam from the enriched uranium, puised spallation source at the intense pulsed neutron source at Argonne National Laboratory has been measured ard found to be 0.0283 for $450-\mathrm{MeV}$ protons. Delayed neutrons produce a sample-dependent background in time-of-flight instruments. Because of the distribution of neutron wavelengths in the prompt pulse, the significance of the delayed neutron contribution to the observed scattering profile is strongly dependent on the wavelength and therefore on the magnitude of the momentum transfer. This effect is demonstrated, and a general correction procedıre in current use for small angle neutron scattering data is described.

\section{Introduction}

The use of fissionable materials as targets in pulsed spallation sources results in, in addition to the prompi pulses, a contribution from delayed neutrons. Certain precursor fission fragments undergo one or more steps of beta decay followed by neutron emission, as has been discussed by Carpenter [1,2]. Energetic photons emitted from beta decaying products of fission and spallation processes can also induce delayed neutrons through $(\gamma, n)$ reactions in such materials as ${ }^{9} \mathrm{Be}$ and ${ }^{2} \mathrm{D}$ which may be near the target. Delay half-lives [3] range from fractions of a second to a minute for precursor fission fragments and from a few seconds to several days for fission product gamma rays interacting with ${ }^{9} \mathrm{Be}$. Since the intense pulsed neutron source (IPNS) at Argonne National Laboratory (ANL) normally operates with a pulse frequency of $30 \mathrm{~Hz}$, the delayed neutrons are emitted at times not correlated with the generation of the prompt pulses. One may assume that the delayed neutrons are moderated and emerge in the collimated beam line with approxinately the same spectral distribution as in the prompt pulses. The result is an essentially steady source of delayed neutroris on which the prompt pulses are superimposed, as represented schematically in fig. 1 .

At the source, the prompt pulse of uninodulated neutrons is only some $300 \mathrm{~ns}$ in width; however, down-

\footnotetext{
* This work was performed under the auspices of the U.S. Department of Energy, Division of Materials Science, Office of Basic Energy Sciences, under Contract W-31-109-ENG-38.
}

stream at a distance $L$ from the source, the pulse has spread out revealing the neutron wavelength distribution. Time-of-flight techniques are used to determine the wavelength of neutrons from the prompt pulse

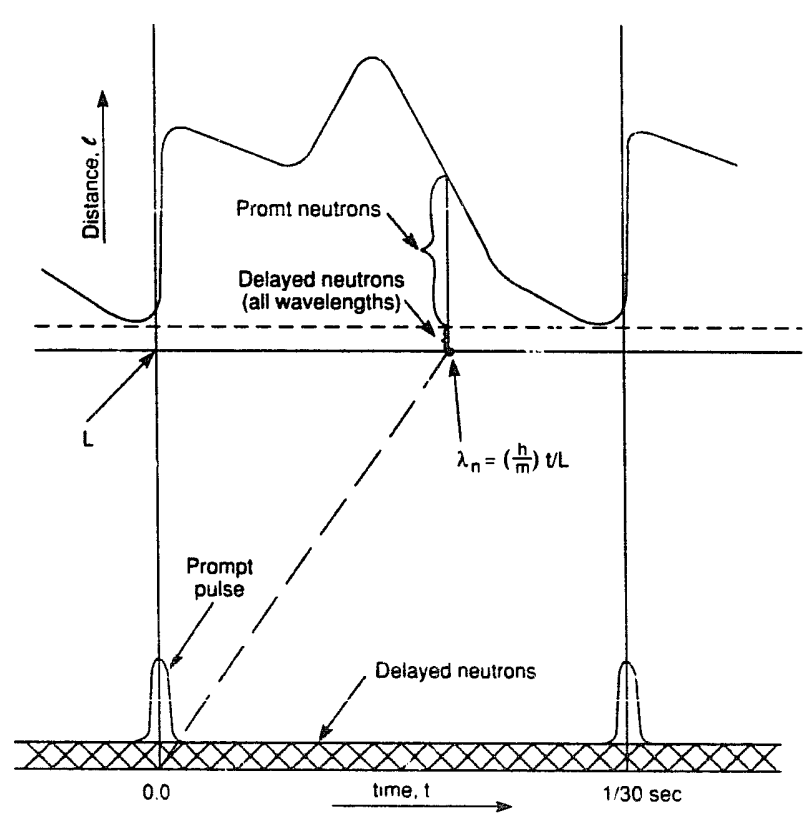

Fig. 1. Schematic representation of the time schedule and intensity distribution at the neutron source (below) and at a distance $L$ from the source (above). Neutrons of energy $E$ $\left(E=\frac{1}{2} m L^{2} / t^{2}\right)$ arrive at position $L$ at time $t$ after generation of the prompt pulse. Delayed neutrons of all energies arrive continuously at $L$. 
scattered by the sample and arriving at the detector at time $t$. Delayed neutrons of all wavelengths are continuously striking the sample, and this contribution scattered by the sample and arriving at the detector is dependent on the specific scattering characteristics of the sample.

The purf -as of this work are to report the determination $o_{1}$ the fraction of delayed neutrons $f_{\mathrm{d}}$ in the $\mathrm{Cl}$ beam line $*$ at the IPNS from the enriched uranium $\left({ }^{235} U\right)$ target, to demonstrate the effect of this delayed neutron contribution on the observed small angle scattering profiles and to describe the general procedure utilized at the small angle diffractometer (IPNS-SAD) to correct for this sample-dependent background. A more comprehensive description of the instrument is given in a forthcoming publication by Epperson et al. [4].

\section{Determination of the fraction of delayed neutrons}

The experimental arrangement used in the determination of the delayed neutron fraction is shown in fig. 2. Fxcept for the absence of a cold $\mathrm{MgO}$ single crystal filter, this represents the upstream end of the $\mathrm{C1}$ beam line on which the IPNS-SAD is Incated, i.e. the section between the neutron source and the beam monitor (M1). Two runs were made; a signal run in which a $0.559-\mathrm{cm}$ thick piece of boron glass (Corning 7749) intercepted the neutron beam before the detector, and a

* The rasult should be independent of the beam in which it is measured and of the presence or absence of an absorber in the beam.

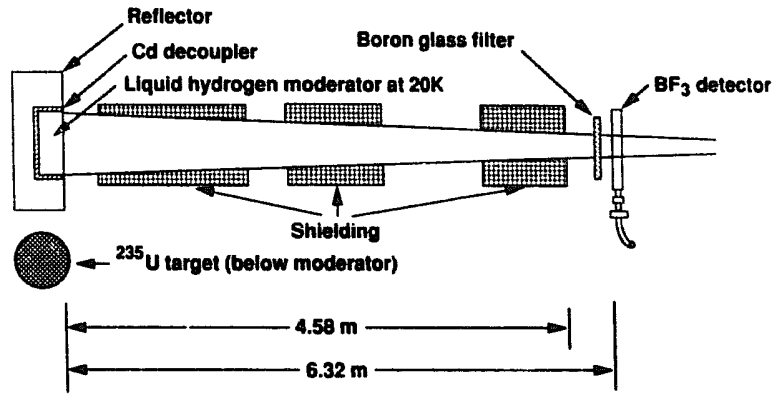

Fig. 2. Experimental arrangement for the source, moderator, boron-glass filter and detector for the delayed neut-on determination.

background run in which a $1.25-\mathrm{cm}$ thick plate of ${ }^{10} \mathrm{~B}$ replaced the boron glass. The purpose of the boron-glass filter was to reduce the counting rate due to the longwavelength neutrons from the prompt pulse, which otherwise would have obscured the delayed neutrons in the latter part of the time frame. Data were collected in 460 equally-spaced time channels corresponding to the wavelength range $0.2 \leq \lambda_{n} \leq 14.0 \AA$, and the data for the signal run are shown in fig. 3. The detector was a low-efficiency $\mathrm{BF}_{3}$ monitor detector. The neutron producing target of IPNS is a water-cooled stack of 77.5\% enriched uranium disks, with $k_{\text {eff }}=0.80$, decoupled from its surroundings by a layer of ${ }^{10} \mathrm{~B}$, which maintains short $(<0.3 \mu \mathrm{s})$ primary neutron pulses. With $450-\mathrm{MeV}$ protons and a time-averaged current of $15 \mu \mathrm{A}$, it produces about $4 \times 10^{15}$ neutrons/s, in pulses at $30 \mathrm{~Hz}$. The moderator serving SAD is of liquid hydrogen at 20 $\mathrm{K}$, in a 0.7 -liter container which has grooves on the viewed surface to increase the neutron beam intensity.

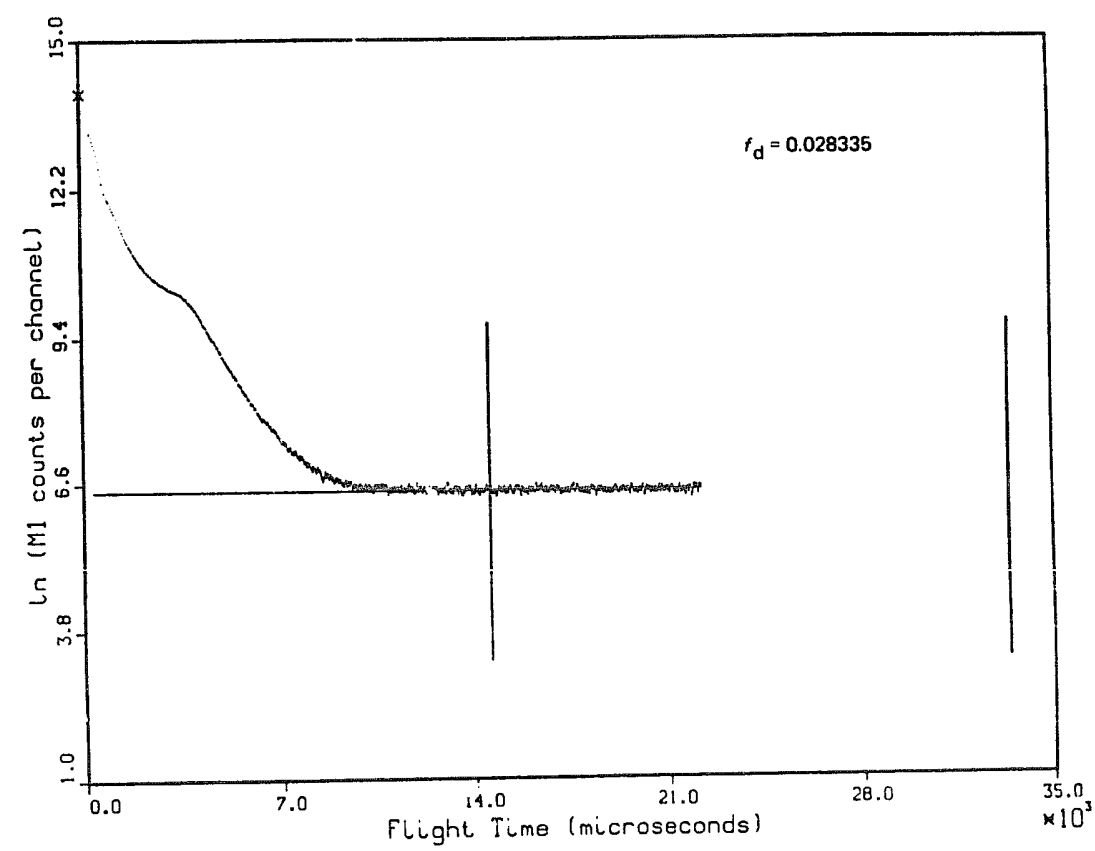

Fig. 3. The distribution of counts in the signal run (RUN 5441); a total of 9004634 counts were recorded within a period of $8.8 \mathrm{~h}$ in 460 equally-spaced time channels over the wavelength range $02 \leq \lambda_{n} \leq 14.0 \AA$. 
The moderator is surrounded by graphite and beryllium as reflector, decoupled by a $0.5-\mathrm{mm}$ layer of cadmium.

A formal presentation of the various contributions to the signal and background runs has been given by Carpenter [2], and it is not necessary to repeat the details here. We choose a data point $N_{\mathrm{p}}$ in the tail of the profile beyond which the accumulated counts per channel is constant; in fig. 3 , this is point 300 and is indicated by the first vertical line. The second vertical line represents the time at which the next pulse is generated $(1 / 30 \mathrm{~s})$. The average number of counts per channel (delayed neutrons) in the range from point 300 to 460 is 627.5 . Let $C_{\mathrm{p}}$ be the total of prompt plus delayed neutrons over the range from point one to $N_{\mathrm{p}}$; $C_{\mathrm{p}}=8.904 \times 10^{6}$. Let $C_{\mathrm{a}}$ be the total of prompt plus delayed neutrons in the region below the first experimental data point. This value is obtained by linear extrapolation to time zero of the data as shown in fig. 3 , using points 1 to $9 . C_{\mathrm{a}}$ is equal to $6.348 \times 10^{6}$. The delayed neutron contribution $C_{\mathrm{bkg}}$ from $t=0$ to point $N_{\mathrm{p}}$ amolints to $0.1922 \times 10^{6}$. Assuming the delayed neutron contribution is constant in time, we find the number of delayed neutrons $C_{\mathrm{d}}$ in this run over the full duty cycle to be $0.4392 \times 10^{6}$. Hence,

$f_{\mathrm{d}}=\frac{C_{\mathrm{d}}}{C_{\mathrm{a}}+C_{\mathrm{p}}-C_{\mathrm{bkg}}+C_{\mathrm{d}}}$.

The value of $f_{\mathrm{d}}$ is thus 0.0283 for the IPNS enriched target *.

\section{Correction of the IPNS-SAD data for delayed neu- trons}

Having an experimental value for the fraction of delayed neutrons in the $\mathrm{Cl}$ beam, it is possible to devise - pi redure, based on the SANS measurement itself, wi: in permits a correction of the scattering data. The ıntensity measured in the area sensitive detector pixel $(x, y)$ in the time interval from $t_{i}$ to $t_{i+1}$ can be represented as

$I\left(x, y, t_{i}\right)=I_{\mathrm{p}}\left(x, y, t_{i}\right)+I_{\mathrm{d}}\left(x, y, t_{i}\right)$,

where $I_{\mathrm{p}}$ represents the contribution from the prompt pulse and $I_{d}$ that from the delayed neutrons. More explicitly, provided the detector response is independent of time,

$$
\begin{aligned}
I_{\mathrm{p}}\left(x, y, t_{i}\right)= & \int_{\lambda\left(t_{t}\right)}^{\lambda\left(t_{i}+1\right)} \int_{i_{i}}^{t_{1+1}} \phi_{\mathrm{p}}(\lambda) S(x, y, \lambda) \\
& \times \in(x, y, \lambda) \delta\left(t-L_{h}^{m} \lambda\right) \mathrm{d} t \mathrm{~d} \lambda,
\end{aligned}
$$

\footnotetext{
* In an earlier measurement when a depleted uranium target was in use at ANL-IPNS, a value of 0.00441 was obtained for $f_{\mathrm{d}}$.
}

where $\phi_{\mathrm{p}}(\lambda)$ is the differential flux of prompt neutrons incident on the sample, $S(x, y, \lambda)$ is the sample scattering function and $\epsilon(x, y, \lambda)$ is the detector efficiency. Ignoring small corrections, the flight time $t$ is directly related to the neutron wavelength $\lambda_{n}$ by

$\lambda_{\mathrm{n}}=(h / m) t / L$,

where $h$ is Planck's constant, $m$ the neutron mass and $L$ is the distance of the detector from the source.

In a similar manner

$$
\begin{aligned}
I_{\mathrm{d}}\left(x, y, t_{i}\right)= & f_{\mathrm{d}} \frac{\Delta t_{i}}{\Delta t} \int_{\lambda\left(t_{i}\right)}^{\lambda\left(t_{i}+1\right)} \int_{t_{\min }}^{t_{\text {max }}} \phi_{\mathrm{p}}(\lambda) S(x, y, \lambda) \\
& \times \in(x, y, \lambda) \delta\left(t-L_{\hbar}^{m} \lambda\right) \mathrm{d} t \mathrm{~d} \lambda,
\end{aligned}
$$

where $f_{\mathrm{d}}$ is the fraction of delayed neutrons in the beam, $\Delta t_{i}$ is the width of the $i$ th time slice and $\Delta t$ is the full time width of the duty cycle. Ideally, $t_{\min }$ and $t_{\max }$ should equal zero and the time to the succeeding pulse, respectively, but, for practical reasons, these are approximated by the minimum and maximum flight times used in the experiment. Although one may choose to analyze scattering data from any desired range of prompt pulse time slices, it should be appreciated that in making the delayed neutron correction, the widest possible flight time range should be utilized. Furthermore, a correction for delayed neutrons must be made for each run associated with a given SANS experiment; typically this set will include the wavelength distribution of neutrons incident on the sample, the wavelength-dependent transmission coefficients and the sample, background and dark current scattering runs. The correction represented by eq. (5) is subtracted pixel by pixel for each time slice during data reduction.

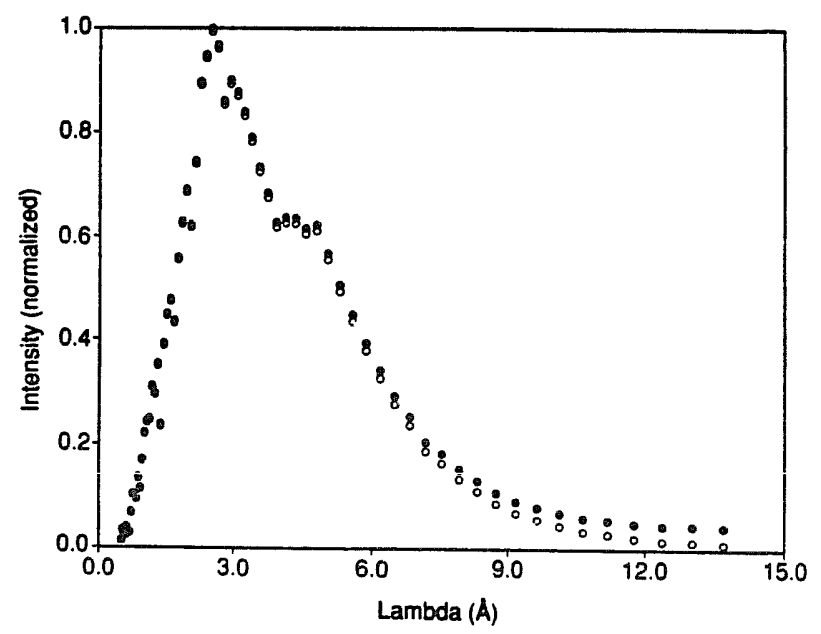

Fig. 4. Wavelength distribution of neutrons in the $\mathrm{Cl}$ beam line at the ANL-IPNS; filled circles are the raw distribution (normalized) measured with $\Delta t / t=0.05$ binning and the open circles are after correction for the delayed neutrons $\left(f_{\mathrm{d}}=\right.$ 0.0283 ). 
The distribution of neutron wavelengths measured directly with the area sensitive detector with a liquid nitrogen cooled $\mathrm{MgO}$ single crystal filter in the beam and constant $\Delta t / t=0.05$ binning is shown in fig. 4 , with and without the correction for delayed neutrons. As can be seen, the delayed neutron correction becomes critical in the long vavelength part of the spectrum from the enriched uranium target. For a given instru- mental geometry, the longer wavelengths contribute to the lower $Q$ range and the shorter wavelengths to the higher $Q$, respectively, and the delayed neutrons can have a substantial effect on the resulting SANS profile. An example is shown in fig. 5, the radially averaged scattering from a polymer sample (Bates poly) which is used at the IPNS-SAD as a secondary intensity standard. This is a polymer melt sample consisting of an
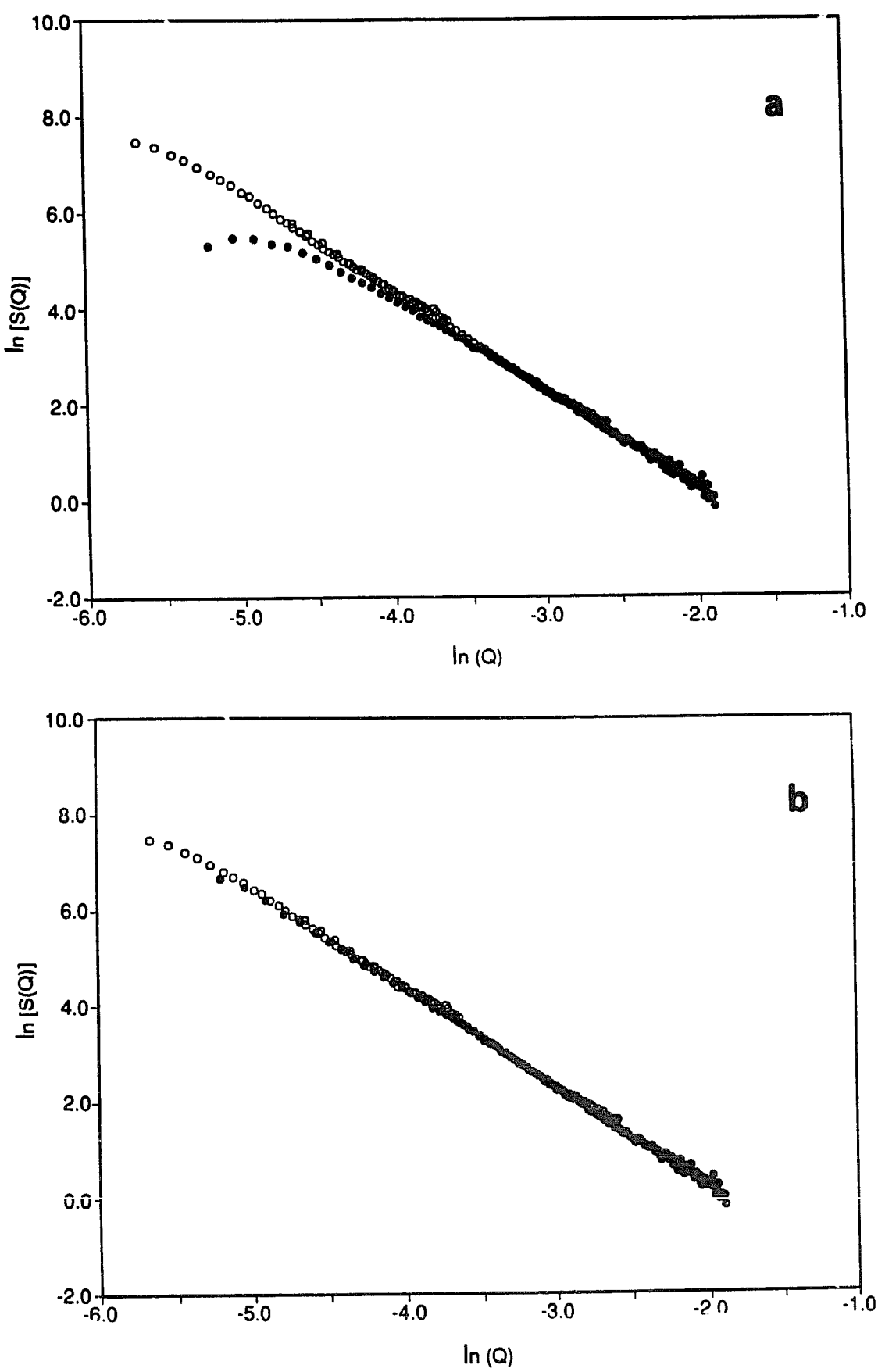

Fig. 5. The effect of correction of IPNS-SAD data for delayed neutrons. Comparison is made with a secondary intensity standard (Bates poly) measured at ORNL; a) no correction of the IPNS-SAD data for delayed neutrons and b) correction of the IPNS-SAD data for $2.83 \%$ delayed neutrons $\left(Q=\left(4 \pi / \lambda_{n}\right) \sin \theta\right)$. 
equal volume mixture of deuterated and protonated high molecular weight polystyrene whose absolute cross section is known from measurements at the steady state neutron source at ORNL [5]. Fig. 5a shows the comparison when no correction has been made to the SAD data for delayed neutrons, and fig. $5 \mathrm{~b}$ after correction, using the experimentally determined value of $f_{\mathrm{d}}$. The correction results in satisfactory agreement of the SAD data with that from ORNL. These data demonstrate convincingly the necessity of making the correction for delayed neutrons when using pulsed spallation neutrons from a fissionable target and the adequacy of the present delayed neutron correction procedure.

\section{Conclusions}

A straighforward measurement allows a determination of the fraction of delayed neutrons in the neutron beam from a pulsed spallation source. For the enriched uranium target now in use at the ANL-IPNS, $f_{\mathrm{d}}$ was found to be 0.0283; for the depleted uranium target previously used, the value was found to be 0.00441 . The effect of the delayed neutrons is to produce a sampledependent background. A procedure using the small angle scattering measurements themselves allows a reasonable correction for the delayed neutrons, and this has been demonstrated on experimental data. The correction procedure is formally the same for any sample scatterer, hence it is appropriate for routine use at a user-oriented facility.

\section{References}

[1] J.M. Carpenter, Nucl. Instr. and Meth. 145 (1977) 91.

[2] J.M. Carpenter, Nucl. Instr. and Meth. 175 (1980) 287.

[3] Reactor Physics Constants, ANL-5800 (1963).

[4] J.E. Epperson, J.M. Carpenter, R.K. Crawford, P. Thiyagarajan and T.E. Klippert, in preparation.

[5] F.S. Bates and G.D. Wignall, Macromol. Rev. 19 (1986) 934. 